\title{
Mutations in Panicle Development Affect Culm Elongation in Rice
}

\author{
Hidehiko Sunohara'), Hikaru Satoh ${ }^{2)}$ and Yasuo Nagato*1) \\ 1) Graduate School of Agricultural and Life Sciences, University of Tokyo, 1-1-1 Yayoi, Bunkyo, Tokyo 113-8657, Japan \\ 2) Graduate School of Genetic Resources Technology, Kyushu University, 6-10-1 Hakozaki, Fukuoka 812-8581, Japan
}

Internode elongation of the rice culm begins almost simultaneously with the change from the vegetative phase to the reproductive phase. Although this synchronism suggests the existence of an interaction between the panicle development and culm internode elongation, little information is available about panicle and internode interaction. In this study, we examined 18 mutants from cv. Taichung 65 that were defective in panicle and/or spikelet development. The 18 mutants were classified into three groups by the organ in which the major mutant phenotypes were observed; (1) mutants with aberrant rachis/branches, (2) mutants with aberrant spikelets/flowers, (3) mutants with aberration in both rachis and spikelets. Most of the mutants with aberrant rachis/branches showed an abnormal culm elongation. In contrast, those defective in only spikelet development showed an almost normal culm elongation. The correlation analysis showed that panicle traits were significantly correlated with the length of the distal one or two internodes. Principal component analysis of the culm internode length showed that the mutants defective in rachis/branch development were positioned far from the wild type, while those with normal panicles and aberrant spikelets were distributed near the wild type. These analyses revealed that culm elongation, especially for the upper internodes, was affected by the early developmental mode of panicles. Although the mechanism by which panicle development regulates the elongation of upper internodes has not yet been identified, the present study indicates that the elucidation of early panicle development is essential for a comprehensive understanding of culm internode elongation.

Key Words: Oryza sativa L., rice, mutant, panicle, culm elongation.

\section{Introduction}

Since plant height is an important agronomical trait in cereals, a large number of studies have been carried out to determine how culm length is genetically regulated. Most of these studies utilized dwarf mutants, such as maize dwarf-1, barley $u z(u z)$, wheat reduced height-1 (rht-1), and so on

Communicated by N. Kurata

Received September 6, 2002. Accepted October 24, 2002.

*Corresponding author (e-mail: anagato@mail.ecc.u-tokyo.ac.jp)
(Nelson 1967, Takahashi and Yamamoto 1951, Gale and Marshall 1976). In rice, more than 60 loci associated with dwarfism have been identified based on recessive mutations (Futsuhara et al. 1986, Kamijima et al. 1996). In normal rice plants, culm elongation occurs at about the time of floral induction for the distal four or five internodes, while the proximal internodes do not elongate. Rice dwarf mutants were categorized based on the elongation pattern of the distal internodes. Takahashi and Takeda (1969) and Takeda (1977) classified dwarf mutants into five types, dn-, dm-, d6-, nland sh-types. In the dn-type, the internode elongation pattern was similar to that of the wild type, i.e. all the internodes were proportionally shortened. In the dm-type, the second internode counted from the top was specifically shortened. In the d6-type, the elongation occurred only in the uppermost internode, and in the nl-type, the elongation of the upper internodes was reduced and the lower internodes elongated. Finally, in the sh-type, elongation of the uppermost internode did not occur. Although these dwarf types except for the dn-type displayed a specific elongation pattern of culm internodes, it remained to be determined how these genes for dwarfism act to induce such specific phenotypes. This categorization is applicable to many dwarf mutants from various cultivars, in spite of the difference in the total number of culm internodes, suggesting that the position of each culm internode can not be determined by counting the number from the base. Instead, elongation of culm internodes can be analyzed when their position is determined from the top, suggesting the possibility of interaction between panicle development and culm internode elongation.

In contrast to a large number of dwarf mutants, few long-culm mutants have been reported (Okuno and Kawai 1978a, 1978b). Although long-culm mutants would be as useful as dwarf mutants from the viewpoint of the regulation of plant height, they have not been fully analyzed.

Recently, several genes for dwarfism that induce a specific elongation pattern of culm internodes have been cloned in rice. The first is the $D 1$ (Daikoku dwarf) locus which encodes the heterotrimeric G protein $\alpha$-subunit (Ashikari et al. 1999, Fujisawa et al. 1999, Fujisawa et al. 2001). Since the D1 protein is distributed in all the internodes, it is unclear how the $d 1$ mutation induces specific dm-type dwarfism. The D6 gene has been found to encode a homeodomain protein OSH15 (Sato et al. 1999). Again, the expression pattern of OSH15 does not explain the d6-type internode elongation pattern. Recently, it has been reported that D61/OsBRI1 gene is an ortholog of BRI1 in Arabidopsis encoding a puta- 
tive BR (brassinosteroid) receptor kinase (Yamamuro et al. 2000). Interestingly, two alleles of D61 showed different internode elongation patterns, dm-type and d6-type (Wu et al. 1999). Together with the expression pattern of $D 61$, we assume that $D 61$ does not directly regulate the elongation of a specific internode. Thus, the mechanism of a specific internode elongation can not be fully explained by the causative gene expression pattern.

In the course of analyzing a number of developmental mutants in rice, we have empirically recognized that mutants defective in panicle development also exhibited aberrant culm elongation, in most cases, dwarfism. As for the temporal relationship between the culm internode elongation and panicle development, Takeda and Takahashi (1973) reported that when the vegetative period was short and a small number of leaves was formed on the culm, culm internode elongation started at the same time as the onset of panicle development, whereas when the vegetative period was long and many leaves were formed on the culm, the internode elongation started independently of panicle initiation. In any case, culm elongation and panicle development are temporally overlapping. Thus, it is suggested that panicle development may interact with the elongation of culm internodes. However, almost no studies have been carried out to determine how panicle and culm interact in the course of development.

To determine how panicle development affects culm elongation, we analyzed 18 mutants showing abnormal rachis/branch and/or spikelet development. It was observed that in the mutants showing abnormal rachis/branch development, culm elongation tended to be affected more significantly than in those showing only abnormal spikelet development.

\section{Materials and Methods}

\section{Plant materials}

We used 18 recessive rice (Oryza sativa L.) mutants showing abnormal panicles and/or spikelets derived from cv. Taichung 65 (Table 1). Four of them, fon1-1 (floral organ number1-1) (Nagasawa et al. 1996, Nagato and Nagasawa 1997), dl-sup1 (drooping leaf-superman1) (Nagato and Nagasawa 1997), ur2 (undulated rachis 2) (Iwata and Omura 1984) and ri (verticillate rachis) (Iwata and Omura 1984, Murai and Iizawa 1994), were reported earlier. The other 14 mutants, fm 2, fm 4, fm 14, fm 20, fm 23, fm 24, fm 55, fm 60, fm 61, TCM2084, TCM2542, TCM2830, TCM2902 and TCM3064, were identified from a $\mathrm{M}_{2}$ population of Taichung 65 subjected to mutagen treatment with methylnitroso-urea, and they were designated by the strain number. They were selected based on their panicle and/or spikelet abnormalities. We used Taichung 65 as the wild type.

\section{Growing conditions of plants}

Homozygous seeds of 17 mutants and the wild type were sown at the end of April. Since only $d l$-sup 1 was completely sterile, seeds set on heterozygous plants were used.
The seedlings were transplanted at the end of May to a paddy field located in the Experimental Farm of the University of Tokyo. Then they were grown under conventional cultural conditions. At the maturity stage, five or more main culms of each mutant were sampled.

\section{Analysis of traits}

As for the rachis and primary branches, we measured the length, the number of internodes and internode length. Also, the number of primary branches and the number of degenerated primary branch primordia were counted. For the spikelets, we observed the number and identity of organs and other abnormalities. We measured the length of each internode of the main culm. As mentioned previously, it is convenient to determine the position of the culm internodes from the top. Then in this study, we designated the internode just below the uppermost one as -1 internode. Similarly, the third, fourth, fifth and sixth internodes counted from the top were designated as $-2,-3,-4$, and -5 internodes, respectively.

\section{Results}

\section{Panicle phenotypes of 18 mutants}

We classified the 18 mutants used in this study into three groups based on the organs showing a major mutant phenotype (Table 1). The first group displayed major defects in the rachis and branch development, the second group in the spikelet development, and the last group in the development of both organs.

The first group showed abnormal rachis and branches but almost normal spikelets, and consisted of ten mutants, $r i$, fm 23, fm 24, fm 60, fm 61, TCM2084, TCM2542, TCM2830, TCM2902 and TCM3064 (Table 1 and Table 2). Nine of the ten mutants, except for fm 24 , had a short rachis (Fig. 1A and Table 2). Short rachis due to the shortening of the rachis internode was found in fm 60, TCM2084 and TCM2542. In TCM2902, the number of rachis internodes was reduced, but not the internode length. Rachis of $\mathrm{fm} 23$, TCM2830 and TCM3064 showed a reduction of both the length and number of internodes. Short rachis in two mutants, $r i$ and fm61, was different from that of the above seven mutants. In fm 61 , the rachis internode was $22 \%$ longer than that of the wild type, and the number of rachis internodes was $57 \%$ smaller than that of the wild type. That is, the excessive elongation of the rachis internodes could not compensate for the decrease in the rachis internode number. The short rachis of $r i$ was characterized by the failure of elongation of several internodes (Fig. 1B). Then two or three branches seemed to develop from one rachis node.

In this group, the other processes of panicle development were also affected. In four mutants, fm 23, TCM2084, TCM2542 and TCM3064, several primary branches in the basal region tended to degenerate (Fig. 1C-G). Primary branches in most mutants of this group were significantly shorter than those in the wild type. In TCM2830, the bracts 
Table 1. List of 18 panicle mutants used in this study

\begin{tabular}{clll}
\hline \hline Group & Mutant & Aberrant organ & \multicolumn{1}{c}{ Major phenotype } \\
\hline 1 & ri & Rachis/branch & Verticillated rachis, short panicle \\
& fm 23 & Rachis/branch & Short panicle, degeneration of some of the basal primary branches \\
& fm 24 & Rachis/branch & Undulated rachis and primary branches \\
fm 60 & Rachis/branch & Short panicle \\
& fm 61 & Rachis/branch & Short panicle \\
& TCM2084 & Rachis/branch & Short panicle, degeneration of some of the basal primary branches \\
& TCM2542 & Rachis/branch & Short panicle, degeneration of some of the basal primary branches \\
& TCM2830 & Rachis/branch & Short panicle, bract elongation \\
& TCM2902 & Rachis/branch & Short panicle \\
& TCM3064 & Rachis/branch & Short panicle \\
\hline \multirow{2}{*}{2} & dl-sup 1 & Spikelet & Conversion of carpel to stamens \\
& fm 2 & Spikelet & Cell mass between stigmas \\
& fm 14 & Spikelet & Increase in the number of stigmas \\
& fm 55 & Spikelet & Increase in the number of glumes and stamens, glume-like lodicule \\
& fon 1-1 & Spikelet, rachis/branch & Increase in the number of stamens and carpels, long panicle \\
& ur2 & Spikelet, rachis/branch & Increase in the number of glumes, undulated rachis and branches, short panicle \\
& fm 4 & Spikelet, rachis/branch & Increase in the number of stamens and carpels, aberrant glume, short panicle \\
& fm 20 & Spikelet, rachis/branch & Increase in the number of primary branches, stamens and carpels \\
\hline
\end{tabular}

Table 2. Panicle traits in 18 mutants

\begin{tabular}{|c|c|c|c|c|c|c|c|c|}
\hline \multirow[b]{2}{*}{ Group } & \multirow[b]{2}{*}{ Mutant } & \multirow[b]{2}{*}{$\begin{array}{l}\text { Rachis length } \\
\text { (mm) }\end{array}$} & \multicolumn{2}{|c|}{ Rachis internode } & \multicolumn{2}{|c|}{ Primary branch } & \multirow{2}{*}{$\begin{array}{c}\text {-No. of degenerated } \\
\text { primary branches }\end{array}$} & \multirow[b]{2}{*}{$\begin{array}{c}\text { No. of } \\
\text { spikelets }\end{array}$} \\
\hline & & & $\begin{array}{l}\text { Mean length } \\
\quad(\mathrm{mm})\end{array}$ & Number & $\begin{array}{l}\text { Length } \\
(\mathrm{mm})\end{array}$ & Number & & \\
\hline \multirow[t]{11}{*}{1} & Wild type (T65) & $168.2 \pm 13.0$ & $16.6 \pm 1.3$ & $10.2 \pm 1.2$ & $67.3 \pm 18.6$ & $11.2 \pm 1.2$ & 0.0 & $141.0 \pm 26.9$ \\
\hline & ri & $137.2 \pm 14.0 *$ & $13.5 \pm 0.6^{* *}$ & $10.2 \pm 1.5$ & $73.5 \pm 14.5$ & $11.0 \pm 1.6$ & $0.2 \pm 0.4$ & $109.2 \pm 13.9^{*}$ \\
\hline & fm 23 & $93.2 \pm 28.6^{* *}$ & $11.8 \pm 1.6^{* *}$ & $8.3 \pm 2.3^{*}$ & $19.5 \pm 2.1 * *$ & $4.0 \pm 2.6^{* *}$ & $5.0 \pm 0.6^{* *}$ & $9.4 \pm 1.5^{* *}$ \\
\hline & $\mathrm{fm} 24$ & $157.3 \pm 13.5$ & $13.9 \pm 0.7 *$ & $11.3 \pm 1.2$ & $41.4 \pm 12.5^{* *}$ & $12.3 \pm 1.2$ & 0.0 & $125.0 \pm 13.9$ \\
\hline & fm 60 & $128.8 \pm 11.5^{* *}$ & $13.4 \pm 1.0 * *$ & $9.6 \pm 0.9$ & $49.6 \pm 4.6^{*}$ & $10.6 \pm 0.9$ & 0.0 & $63.3 \pm 6.7^{* *}$ \\
\hline & $\mathrm{fm} 61$ & $88.6 \pm 14.0^{* *}$ & $20.3 \pm 1.8^{* *}$ & $4.4 \pm 0.9^{* *}$ & $41.1 \pm 9.2 * *$ & $5.4 \pm 0.9 * *$ & 0.0 & $33.4 \pm 9.3 * *$ \\
\hline & TCM2084 & $114.6 \pm 6.7 * *$ & $9.0 \pm 1.7 * *$ & $13.0 \pm 1.9$ & $25.8 \pm 7.3 * *$ & $9.0 \pm 1.2 *$ & $5.0 \pm 1.7 * *$ & $45.0 \pm 5.2^{* *}$ \\
\hline & TCM2542 & $97.2 \pm 11.2^{* *}$ & $8.7 \pm 1.4^{* *}$ & $11.5 \pm 2.7$ & $27.1 \pm 9.2 * *$ & $11.3 \pm 2.3$ & $1.2 \pm 0.4^{* *}$ & $53.5 \pm 6.3 * *$ \\
\hline & TCM2830 & $59.6 \pm 10.1^{* *}$ & $10.5 \pm 1.4 * *$ & $5.8 \pm 1.6^{* *}$ & $34.1 \pm 4.8^{* *}$ & $6.8 \pm 1.6^{* *}$ & 0.0 & $36.2 \pm 8.2 * *$ \\
\hline & TCM2902 & $79.5 \pm 18.0^{* *}$ & $16.7 \pm 2.2$ & $4.8 \pm 1.0^{* *}$ & $35.0 \pm 6.7 * *$ & $5.5 \pm 0.6^{* *}$ & $0.3 \pm 0.4$ & $30.0 \pm 2.9^{* *}$ \\
\hline & TCM3064 & $54.6 \pm 18.5^{* *}$ & $10.3 \pm 2.9^{* *}$ & $5.3 \pm 0.5^{* *}$ & $35.0 \pm 8.8^{* *}$ & $4.1 \pm 1.2 * *$ & $2.1 \pm 1.0^{* *}$ & $21.1 \pm 7.6^{* *}$ \\
\hline \multirow[t]{4}{*}{2} & $d l$-sup 1 & $203.4 \pm 17.8^{* *}$ & $17.8 \pm 1.1$ & $11.4 \pm 0.5$ & n.m. & $11.6 \pm 1.1$ & $0.8 \pm 0.7$ & $122.2 \pm 29.7$ \\
\hline & $\mathrm{fm} 2$ & $156.4 \pm 17.7$ & $17.9 \pm 0.4$ & $9.0 \pm 0.0$ & n.m. & $10.0 \pm 0.0$ & $0.0 \pm 0.0$ & $111.6 \pm 38.7$ \\
\hline & fm 14 & $154.2 \pm 9.2$ & $13.4 \pm 0.9 * *$ & $11.6 \pm 1.5$ & n.m. & $12.0 \pm 1.0$ & $0.6 \pm 0.8$ & $116.4 \pm 14.4$ \\
\hline & fm 55 & $137.2 \pm 26.9$ & $17.5 \pm 1.0$ & $7.5 \pm 3.5$ & $58.5 \pm 13.4$ & $8.5 \pm 3.5$ & 0.0 & $124.4 \pm 35.3$ \\
\hline \multirow[t]{4}{*}{3} & fon $1-1$ & $213.4 \pm 18.9^{* *}$ & $10.9 \pm 1.2 * *$ & $19.8 \pm 3.1^{* *}$ & $64.6 \pm 19.6$ & $20.8 \pm 3.1^{* *}$ & 0.0 & $230.0 \pm 39.1^{* *}$ \\
\hline & $u r 2$ & $107.8 \pm 3.6^{* *}$ & $7.4 \pm 0.7 * *$ & $14.8 \pm 1.5^{* *}$ & $33.9 \pm 4.6^{* *}$ & $15.8 \pm 1.5^{* *}$ & 0.0 & $89.8 \pm 23.0^{* *}$ \\
\hline & $\mathrm{fm} 4$ & $125.0 \pm 27.3^{*}$ & $16.4 \pm 4.2$ & $7.5 \pm 1.0^{* *}$ & n.m. & $7.8 \pm 1.9^{* *}$ & $0.6 \pm 0.7$ & $82.0 \pm 3.6^{* *}$ \\
\hline & $\mathrm{fm} 20$ & $193.0 \pm 25.6$ & $9.4 \pm 0.8^{* *}$ & $20.4 \pm 2.7^{* *}$ & n.m. & $21.2 \pm 3.0^{* *}$ & $0.2 \pm 0.4$ & $113.6 \pm 65.5$ \\
\hline
\end{tabular}

\pm : Standard deviation

$*, * *$ : significantly different from the wild type at $5 \%$ and $1 \%$ levels, respectively.

n.m.: not measured

of the primary branches were frequently overdeveloped (Fig. $1 \mathrm{H})$, which usually degenerated at the early stage.

The remaining mutant of this group, fm 24 , had no short panicle. The fm 24 showed an undulation of the rachis and branches (Fig. 1J), unlike the wild type which displayed a straight elongation.

The second group consisted of four mutants, dl-sup1, fm 2, fm 14 and fm 55 (Table 1 and Fig. 2). The dl-sup 1 mu- tant showed a homeotic conversion of the pistil to stamens, in other words, several stamens were produced in the region where a carpel should be present (Fig. 2B). In two mutants, $\mathrm{fm} 14$ and fm 55, the number of floral organs increased. In fm 14, the number of stigmas increased from two to three or four, while the number of the other organs was normal (Fig. 2C). In fm 55, lodicules frequently became glume-like (Fig. 2D). In addition, the numbers of lemmas and stamens 


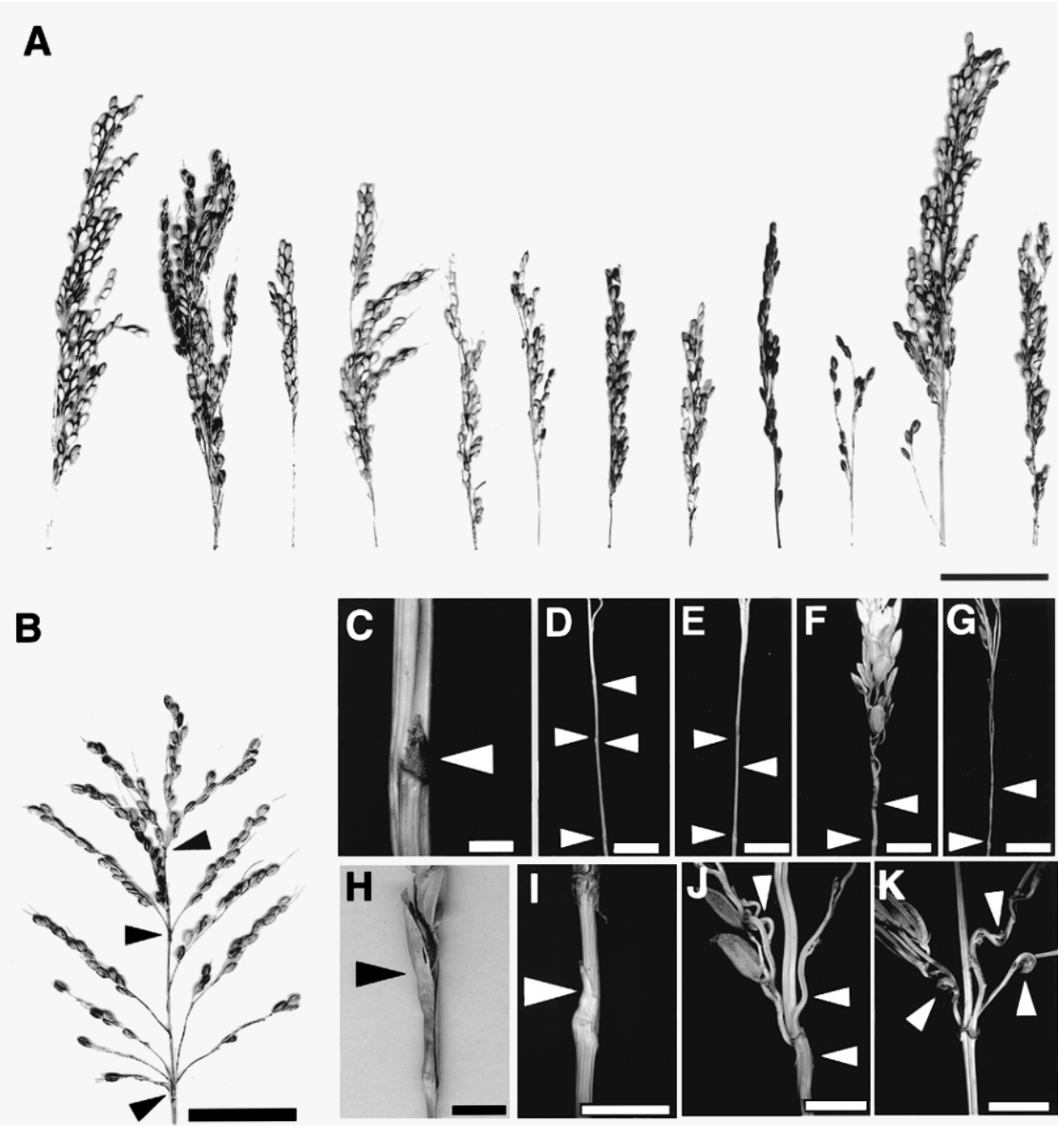

Fig. 1. Phenotypes of mutants showing abnormal rachis/branches. (A) Panicles of 11 mutants showing abnormal rachis length. From left to right, wild type (Taichung 65), fm 23, fm 60, fm 61, TCM2084, TCM2542, TCM2830, TCM2902, TCM3064, fonl-1 and ur2. (B) Panicle of $r i$ forming several primary branches in a cluster (arrowheads). (C) Aborted branch primordium (arrowhead) in the basal region of $\mathrm{fm} 23$ rachis. (D) to (G) Aborted branch primordia (arrowheads) in $\mathrm{fm} \mathrm{23}$, TCM2084, TCM2542 and TCM3064, respectively. (H) and (I) Overdeveloped bracts (arrowheads) in TCM2830 and $u r 2$, respectively. $(\mathrm{J})$ and $(\mathrm{K})$ Undulation of rachis and primary branches (arrowheads) in fm 24 and $u r 2$, respectively. Bars $=5 \mathrm{~cm}$ in (A) and (B), $1 \mathrm{~mm}$ in (C), $1 \mathrm{~cm}$ in (D) to (G), $(\mathrm{J})$ and $(\mathrm{K})$, and $5 \mathrm{~mm}$ in $(\mathrm{H})$ and $(\mathrm{I})$.

increased (Fig. 2H). The fm 2 mutant showed a cell mass between two stigmas. Based on the longitudinal sections, the cell mass in fm 2 was derived from the excessive proliferation of integument (Fig. 2L). Panicle traits in this group were almost normal. As shown in Table 2, the rachis in $\mathrm{dl}$-sup1 was long, whereas the rachis internode in fm 14 was short. However, other panicle traits (number of rachis internodes, primary branch length, number of primary branches, number of spikelets, and so on) were almost normal. Also, the number of elongating rachis internodes in fm 14 and $d l$-supl was the same as that in the wild type. Thus, the panicles of fm 14 and $d l$-supl were almost normal.

The last group consisted of four mutants, fon 1-1, ur2, fm 4 and fm 20 (Table 1). Mutants in this group exhibited ab- normalities in both rachis and spikelets. A significantly aberrant rachis length was observed in fon 1-1, ur2 and fm 4 (Fig. 1A). The fon 1-1 mutant had a long rachis, and $u r 2$ and fm 4 had a short rachis. The long rachis of fon 1-1 was mainly due to the large number of internodes, which compensated for the reduction associated with short internodes (Table 2). The fon 1-1 mutant was mainly characterized by the increase in the number of floral organs (Fig. 2E). In fon 1-1, the number of pistils increased from one to two or three, and stamens from six to seven, while the number of lodicules seldom increased (Nagasawa et al. 1996). The fm 20 mutant showed similar phenotypes of spikelets to those of fon 1-1 (Fig. 2F). Although the rachis was not appreciably longer, it was composed of a significantly larger number of short internodes 

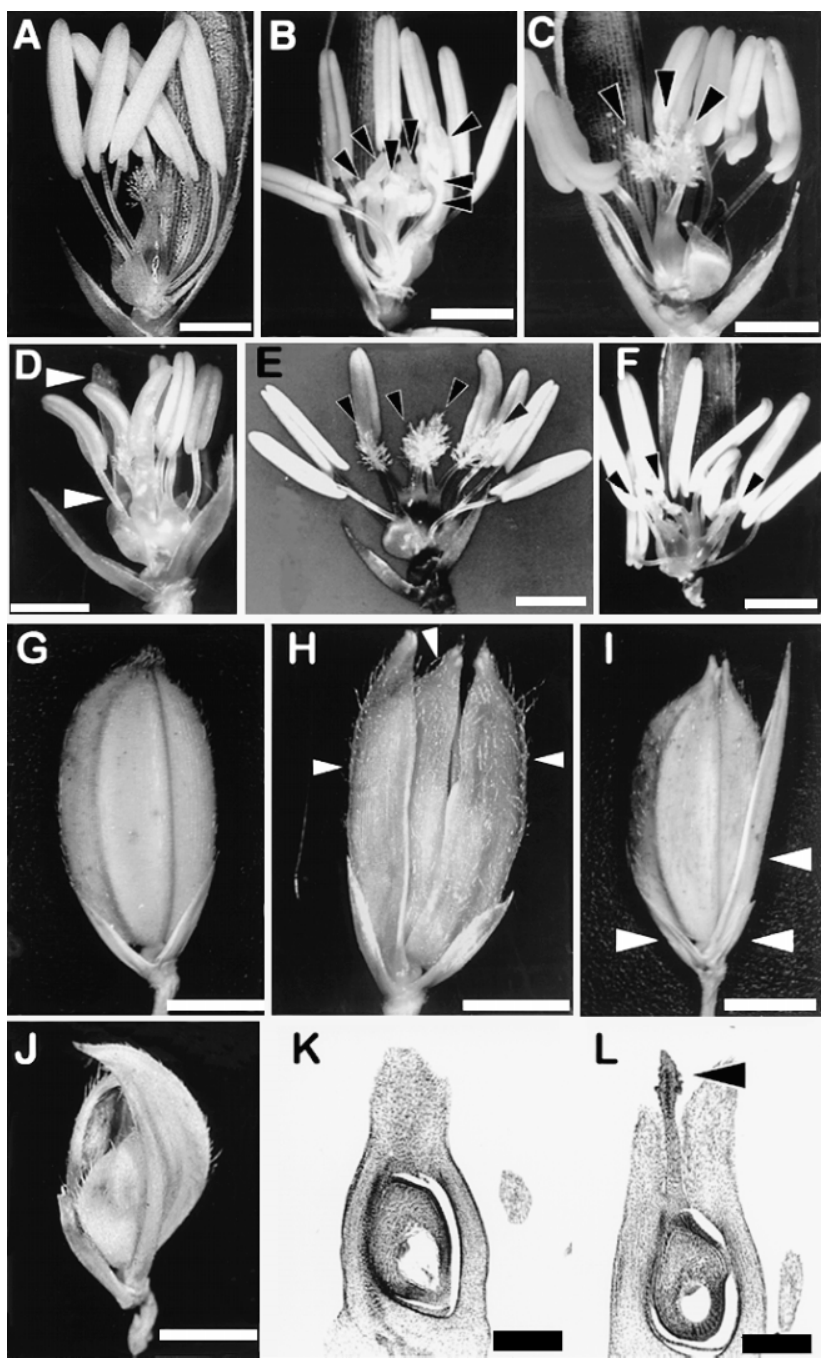

L

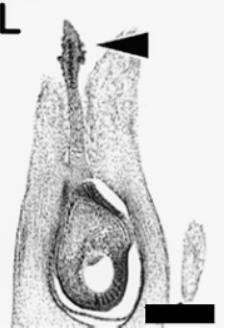

Fig. 2. Phenotypes of mutants showing abnormal spikelet/flower. (A) Wild-type spikelet. (B) $d l$-sup 1 flower showing ectopic stamens converted from carpel (arrowheads). (C) fm 14 flower showing an increase in the number of pistils. (D) fm 55 flower showing glume-like lodicules. (E) fon 1-1 flower showing an increase in the number of stamens and pistils (arrowheads). (F) fm 20 flower showing an increase in the number of stamens and pistils (arrowheads). (G) Wild type spikelet. $(\mathrm{H}) \mathrm{fm} 55$ showing an increase in the number of lemmas (arrowheads). (I) Spikelet of $u r 2$ showing an increase in the number of glumes (arrowheads). (J) Spikelet of fm 4 showing malformed lemma and palea. (K) Longitudinal section of wild type pistil. (L) Longitudinal section of fm 2 pistil showing a cell mass (arrowhead) between two stigmas. Bars $=2.5 \mathrm{~mm}$ in (A) to $(\mathrm{F}), 2 \mathrm{~mm}$ in $(\mathrm{G})$ to $(\mathrm{J})$, and $0.2 \mathrm{~mm}$ in $(\mathrm{K})$ and $(\mathrm{L})$.

(Table 2). In $u r 2$, the rachis was significantly short due to the reduction of the internode length in spite of the increase in the internode number (Table 2). Moreover in $u r 2$, bracts at the basal nodes of the panicle were frequently developed (Fig. 1I), rachis and primary branches were undulated (Fig. $1 \mathrm{~K}$ ), and the number of glumes increased (Fig. 2I). In fm 4, the rachis was composed of a smaller number of internodes (Table 2), and the glume was malformed (Fig. 2J). In flowers of fm 4, the numbers of stamens and carpels increased as in the case of fon 1-1 and fm 20.

\section{Culm elongation}

We measured the culm internode length (Fig. 3). Abnormal culm elongation was detected in 11 mutants, $r i, u r 2$, fm 4, fm 23, fm 24, fm 60, fm 61, TCM2084, TCM2542, TCM2830 and TCM3064 (Fig. 3). The ri and fm 60 mutants had a long culm, and the others had a short culm. In $r i$ and fm 60 , the internodes except for the uppermost one were longer than those in the wild type. In the nine mutants with a short culm, the reduction was remarkable in the uppermost and -1 internodes, while the length of the lower internodes was comparable to that of the wild type. In seven mutants, TCM2902, dl-sup1, fm 2, fm 14, fm 55, fon1-1 and fm 20, the culm length was almost normal. In TCM 2902, although the culm length was normal, the internode elongation pattern was abnormal in that the uppermost and -1 internodes were shorter while the $-3 \sim-5$ internodes became longer. It was observed that all the mutants with an abnormal culm length or internode elongation pattern showed defects in rachis/ branch development.

\section{Relation between panicle development and culm elongation}

Next, to determine how the panicle development was related to culm elongation, we performed a correlation analysis using the data of 18 mutants (Table 3 ). Among the culm internodes, a positive and significant correlation was recognized between two adjacent internodes, while no correlation was observed between two internodes separated by two or more internodes. As for the relation between panicle traits and culm length, the rachis length, primary branch length, number of primary branches and number of spikelets were significantly correlated positively with the length of the uppermost and -1 internodes. The rachis internode length and the number of rachis internodes were significantly correlated with only the uppermost internode length. Thus, panicle development appeared to be related to the elongation of the distal one or two internodes.

To compare temporally the early stages of panicle development and culm elongation, we made longitudinal sections of the shoot apex at approximately the time of the onset of reproductive phase of Taichung 65 . Before the bract of the first primary rachis branch was initiated, the -3 and lower internodes had already started elongating (data not shown). The onset of reproductive development appeared to coincide with the time of initiation of -2 internode elongation (data not shown). That is, the elongation of the distal two internodes occurred during early panicle development (rachis elongation and primary branch differentiation), suggesting that the panicle development affects culm (distal two internodes) elongation.

To determine how the internode elongation pattern is associated with the three groups of 18 mutants, principal component analysis was carried out based on the culm internode length (Fig.4). The first principal component accounting 


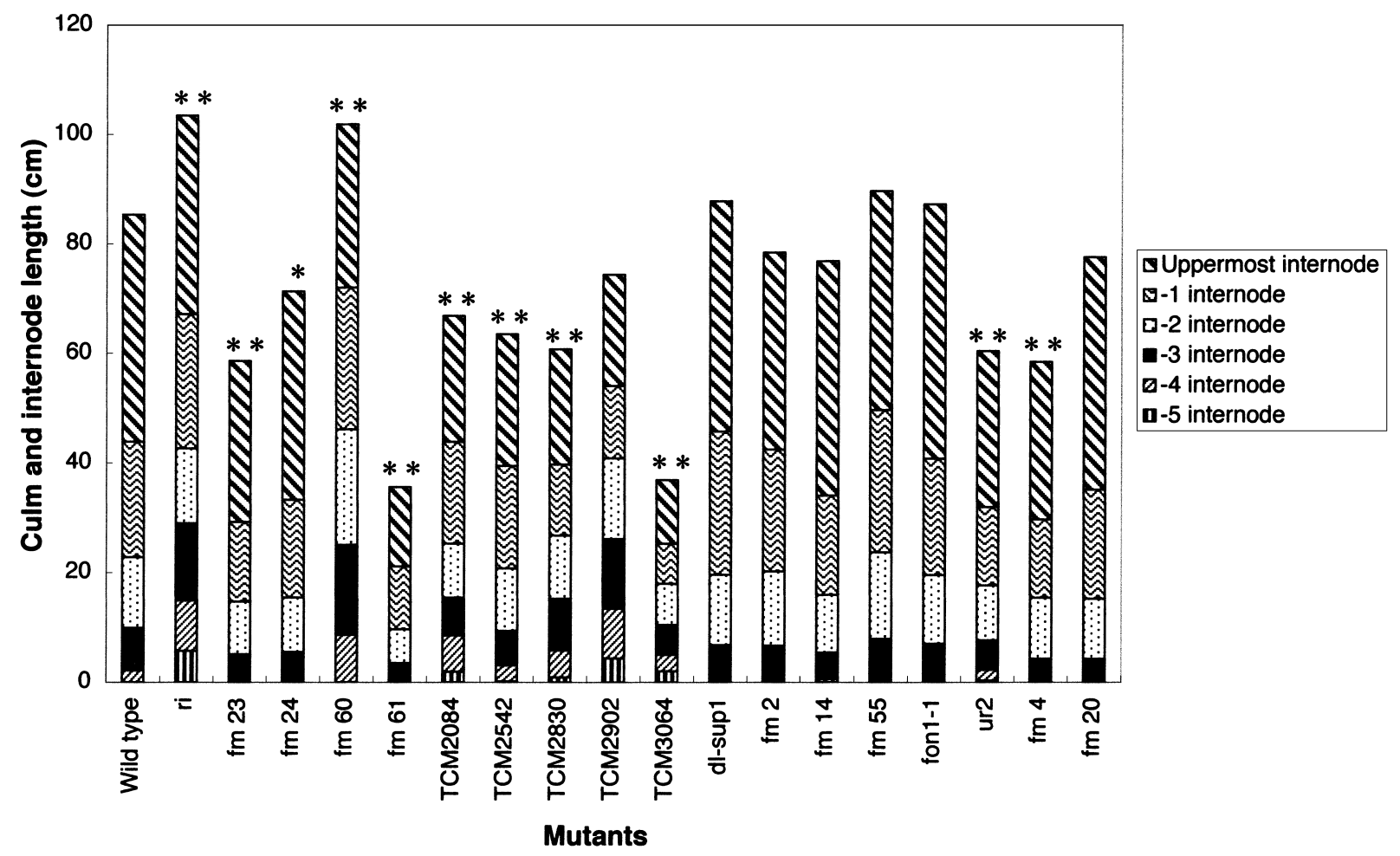

Fig. 3. Culm and internode length in 18 mutants.

*, **: Culm length is significantly different from that of the wild type at $5 \%$ and $1 \%$ levels, respectively.

Table 3. Correlation coefficients between culm internode length and panicle traits in 18 mutants

\begin{tabular}{|c|c|c|c|c|c|c|}
\hline Traits & -5 internode & -4 internode & -3 internode & -2 internode & -1 internode & $\begin{array}{l}\text { Uppermost } \\
\text { internode }\end{array}$ \\
\hline-5 internode length & - & & & & & \\
\hline-4 internode length & $0.77 * *$ & - & & & & \\
\hline-3 internode length & $0.57^{*}$ & $0.86^{* *}$ & - & & & \\
\hline-2 internode length & 0.10 & $0.46^{*}$ & $0.80 * *$ & - & & \\
\hline-1 internode length & -0.09 & 0.06 & 0.41 & $0.71 * *$ & - & \\
\hline Uppermost internode length & -0.28 & -0.37 & -0.02 & 0.36 & $0.74 * *$ & - \\
\hline Rachis length & -0.32 & -0.39 & -0.11 & 0.27 & $0.72 * *$ & $0.92 * *$ \\
\hline Rachis internode length & 0.30 & 0.00 & 0.22 & 0.26 & 0.40 & $0.48^{*}$ \\
\hline No. of panicle internodes & -0.24 & -0.20 & -0.20 & 0.02 & 0.40 & $0.59 * *$ \\
\hline Primary branch length & 0.19 & 0.07 & 0.40 & 0.44 & $0.64^{*}$ & $0.68 * *$ \\
\hline No. of primary branches & -0.23 & -0.20 & -0.11 & 0.14 & $0.46^{*}$ & $0.65 * *$ \\
\hline No. of spikelets & -0.18 & -0.25 & -0.07 & 0.19 & $0.57 *$ & $0.77 * *$ \\
\hline
\end{tabular}

$*, * *$ : significant at $5 \%$ and $1 \%$ levels, respectively.

for $50 \%$ of the total variance mainly reflected the lower internode length, while the second principal component accounting for $37 \%$ of the total variance reflected the distal internode length. The scatter diagram along the first and second principal components revealed that mutants defective in floral organs tended to be distributed in the vicinity of the wild type, while mutants with abnormalities in the rachis and/or branches were scattered far away from the wild type. Moreover, the mutants with abnormalities in both the panicle and spikelets were located between the above two groups. These results indicate that the early panicle development, namely rachis internode and primary branch develop- ment, is strongly associated with the culm internode elongation.

\section{Discussion}

To analyze the relationship between panicle development and culm internode elongation, we used 18 mutants with abnormal reproductive organs. The correlation analysis between panicle traits and culm internode length revealed that the panicle traits were positively correlated with the length of the upper one or two internodes. Moreover, the onset of reproductive development coincided with the initi- 


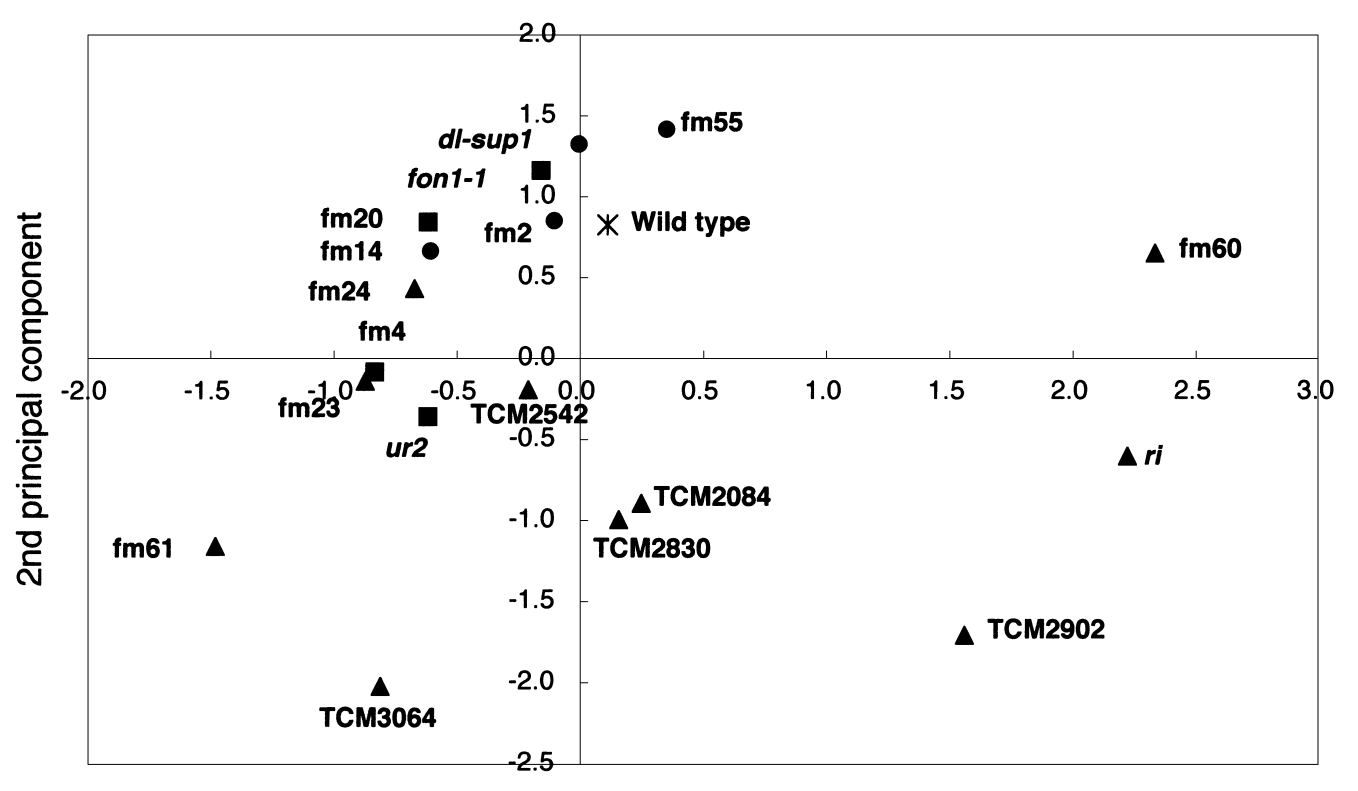

1st principal component

Fig. 4. Scatter diagram of 18 mutants based on the principal component analysis of internode length. * : Wild type, $\boldsymbol{\Delta}$ : Mutants defective in rachis/branches, $\mathbf{0}$ : Mutants defective in spikelets/flowers, $\mathbf{\square}$ : Mutants defective in both organs.

ation of -2 culm internode elongation in Taichung 65. In other cultivars, it was reported that the panicle and -1 internode elongated synchronously (Samoto et al. 1965, Oka and Morishima 1968). Also, the elongation pattern of several upper internodes showing a geometric relation from the top was different from that of the lower internodes showing an identical limited elongation (Takeda and Takahashi 1973). These facts strongly suggest that the mode of panicle development plays an important role in the culm internode elongation. Up to now, however, almost no studies had been carried out from the viewpoint of panicle-culm interaction.

As for the panicle-culm interaction, the examination of culm traits and principal component analysis revealed that defects in spikelets/flowers such as the increase in the number of floral organs and homeotic conversion of floral organs did not affect the culm elongation pattern. Culm elongation is markedly modified in the mutants showing abnormalities at the early stages of panicle development, namely the formation of primary branches and rachis elongation. In considering the relationship between panicle development and culm elongation, it is noteworthy that five out of six mutants, which had a smaller number of primary branches (rachis internodes) resulting in short panicles, were dwarf plants. Since the fewer primary branches in the five mutants were caused by the early abortion of the rachis meristem (unpublished data), the time at which the abortion of the rachis meristem occurs is likely to be an important regulator of culm elongation, especially the elongation of the upper two internodes. TCM2902 is an exceptional mutant characterized by a small number of primary branches and short panicles but by a normal culm length. However, the elongation pattern of the culm internodes of TCM2902 was considerably modi- fied with a reduction of the upper two internodes and excessive elongation of the lower four internodes. In this case, the timing of rachis meristem abortion could have affected the elongation pattern of the culm internodes. There may be an another mechanism whereby panicle development affects culm elongation. For example, two short panicle mutants, $r i$ and fm 60 , showed a long culm. These mutants had a normal number of primary branches but the rachis internodes were short. In the culm of these mutants, the upper two internodes were almost normal, while the lower internodes were excessively long. Thus these mutations may affect culm elongation differently from the other mutations causing short panicles. In contrast to the short-panicle mutants, two mutants, $d l$-supl and fon 1-1, showed long panicles, but their culm length and internode elongation pattern were normal. Thus excessive elongation of the rachis or excessive production of primary branches may not affect culm elongation.

Three mutants, TCM2084, TCM2902 and TCM3064, had short panicles and showed an increase in the number of elongating culm internodes, i.e. -5 internode significantly elongated. Their culms were characterized by the shortening of the uppermost internode and the elongation of -4 and -5 internodes, although the culm length was different among the three mutants, short in TCM2084 and TCM3064 but normal in TCM2902. What causes the increase in the number of elongating internodes and excessive elongation of lower internodes? Yokoo et al. (1980) observed that a late-maturing allele of $L m$ induced a delay in heading time as well as a long culm. Moreover, Yokoo and Okuno (1981) showed that the difference in culm length among the alleles of $\mathrm{Lm}$ was not caused by the upper internodes but by the changes in the number and length of lower elongating internodes. Also 
TCM2084, TCM3064 and TCM2902 were late-heading by more than one week (data not shown). Thus, it is possible that the delay in heading time is associated with the increase in the number of elongating culm internodes.

Among the 18 mutants used in this experiment, nine mutants were dwarf. Four of the nine mutants, fm 23, fm 24, ur 2 and fm 4, showed a dn-type dwarfism. One mutant, TCM3064 was a nl-type dwarf. But the dwarfism of the remaining four mutants, TCM2084, TCM2542, TCM2830 and $\mathrm{fm} 61$, was not classified into any of the five types previously described (Takahashi and Takeda 1969, Takeda 1977). The length of the uppermost internodes of three mutants, TCM2084, TCM2542 and TCM2830, was reduced to about half of the length of the wild type, and the other internodes were not affected, except for TCM2084, in which -5 internode significantly elongated. In fm 61 , the length of the uppermost internode was markedly reduced and the lower internodes were also shortened to some extent while the -4 internode did not elongate. Thus the internode elongation pattern of the 18 mutants was variable and could not necessarily be classified into the known five types, suggesting that a large number of factors were involved in the internode elongation of the culm.

In this study, we used 18 mutants with the common genetic background of $\mathrm{cv}$. Taichung 65 , and revealed that early panicle development exerted a significant effect on the culm internode elongation. To date, dwarfism of culm had been exclusively investigated from the viewpoint of internode elongation, independently of panicle development. Although in $d 1$ and other mutants, both culm and panicle are known to be pleiotropically affected (Konishi 1968, Kawai and Narahari 1971, Ashikari et al. 1999, Fujisawa et al. 1999, Yamamuro et al. 2000), almost no studies have been focused on the interaction between panicle development and culm elongation. Although the mechanism by which panicle development affects culm elongation remains to be identified, the elucidation of early panicle development is essential for a comprehensive understanding of culm internode elongation.

\section{Acknowledgments}

We thank Noboru Washizu, Ken-Ichiro Ichikawa and Chihiro Sasaki (University of Tokyo) for their assistance in cultivating mutant plants in the Experimental Farm, University of Tokyo. This work was supported in part by a Grantin-Aid for Scientific Research on Priority Areas (No. 140367206) from the Ministry of Education, Science and Culture of Japan.

\section{Literature Cited}

Ashikari,M., J.Wu, M.Yano, T.Sasaki and A.Yoshimura (1999) Rice gibberellin-insensitive dwarf mutant gene Dwarf 1 encodes the $\alpha$-subunit of GTP-binding protein. Proc. Natl. Acad. Sci. USA 96: 10284-10289.
Fujisawa,Y., H.Kato and Y.Iwasaki (2001) Structure and function of heterotrimeric G proteins in plants. Plant Cell Physiol. 42: 789794.

Fujisawa, Y., T. Kato, S. Ohki, A. Ishikawa, H. Kitano, T. Sasaki, T.Asahi and Y.Ishikawa (1999) Suppression of the heterotrimeric $\mathrm{G}$ protein causes abnormal morphology, including dwarfism, in rice. Proc. Natl. Acad. Sci. USA 96: 7575-7580.

Futsuhara, Y., F.Kikuchi and J.N.Rutger (1986) Gene symbols for dwarfness. Rice Genet. Newsl. 3: 8-10.

Gale,M.D. and G.A.Marshall (1976) The chromosome location of Gai 1 and Rht 1 genes for gibberellin insensitivity and semi-dwarfism, in a derivative of Norin 10 wheat. Heredity 37: 283-289.

Iwata, N. and T.Omura (1984) Studies on the trisomics in rice plants (Oryza sativa L.) VI. An accomplishment of a trisomic series in japonica rice plants. Jpn. J. Genet. 59: 199-204.

Kamijima,O., T.Tanisaka and T.Kinoshita (1996) Gene symbols for dwarfness. Rice Genet. Newsl. 13: 19-25.

Kawai,T. and P.Narahari (1971) Pattern of reduction of internode lengths and changes of some other characters in short-culm mutants in rice. Indian J. Genet. Plant Breed. 31: 421-441.

Konishi,T. (1968) Studies on EMS-induced mutations in barley. II. Induction of dwarf mutations and characteristics of their mutants. Nogaku Kenkyu 52: 99-109 (in Japanese).

Murai,M. and M.Iizawa (1994) Effects of major genes controlling morphology of panicle in rice. Breed. Sci. 44: 247-255.

Nagasawa, N., M.Miyoshi, H.Kitano, H.Satoh and Y.Nagato (1996) Mutations associated with floral organ number in rice. Planta 198: 627-633.

Nagato,Y. and N.Nagasawa (1997) Mutational dissection of flower development in rice. Gamma Field Symp. 36: 13-25.

Nelson, O.E.J. (1967) Biochemical genetics of higher plants. Ann. Rev. Genet. 1: 245-268.

Oka,H.-I. and H.Morishima (1968) Analysis of genetic variations in plant type of rice. IV. General growth rate, oscillating growth and allometric pattern. Jpn. J. Genet. 43: 191-201.

Okuno,K. and T.Kawai (1978a) Variations of internode length and other characters in induced long-culm mutants of rice. Jpn. J. Breed. 28: 243-250.

Okuno,K. and T.Kawai (1978b) Genetic analysis of induced longculm mutants in rice. Jpn. J. Breed. 28: 336-342.

Samoto,K., H.Suga, K.Sugimoto and K. Suzuki (1965) Studies on the ecological variations of the growth of rice plant caused by the shifting of cultivation season. Bull. Tokai-Kinki Agr. Exp. Sta. 12: 27-38 (in Japanese with English summary).

Sato, Y., N. Sentoku, Y. Miura, H. Hirochika, H. Kitano and M. Matsuoka (1999) Loss-of-function mutations in the rice homeobox gene $\mathrm{OSH} 15$ affect the architecture of internodes resulting in dwarf plants. EMBO J. 18: 992-1002.

Takahashi,R. and J.Yamamoto (1951) Studies on the classification and the geographical distribution of the Japanese barley varieties III. On the linkage relation and the origin of "uzu" or semibrachytic character in barley. Ber. Ohara Inst. landw. Forsch. 9: 399-410.

Takahashi,M.-E. and K.Takeda (1969) Type and grouping of internode pattern in rice culm-Genetical studies on rice plant, XXXVII-. Mem. Fac. Agrc. Hokkaido Univ. 7: 32-43 (in Japanese with English summary).

Takeda,K. (1977) Internode elongation and dwarfism in some gramineous plants. Gamma Field Symp. 16: 1-18.

Takeda,K. and M.-E.Takahashi (1973) Studies on the pattern of organ development in the rice plant. II. Internode distribution pattern 
in relation of number to elongated internodes. Jpn. J. Breed. 23: 7-14.

Wu,X., Y.Ihara, K.Takeda and H.Kitano (1999) New dm-type dwarf mutants varying in internode elongation patterns are controlled by different mutant genes at same locus in rice (Oryza sativa L.). Breed. Sci. 49: 147-153.

Yamamuro,C., Y.Ihara, X.Wu, T.Noguchi, S.Fujioka, S.Takatsuto, M.Ashikari, H.Kitano and M.Matsuoka (2000) Loss of function of a rice brassinosteroid insensitive 1 homolog prevents internode elongation and bending of the lamina joint. Plant Cell 12: 1591-1605.

Yokoo,M. and K.Okuno (1981) Pleitropic effect on internode elongation of alleles conferring heading time of rice. Bull. Natl. Inst. Agr. Sci. D 32: 1-14 (in Japanese with English summary).

Yokoo,M., F.Kikuchi, A.Nakane and H.Fujimaki (1980) Genetical analysis of heading time by aid of close linkage with blast resistance in rice. Bull. Natl. Inst. Agr. Sci. D 31: 95-126 (in Japanese with English summary). 